\title{
Analysis of the Behavior of a Square Plate in Free Vibration by FEM in Ansys
}

\author{
Pascal Kuate Nkounhawa', Dieunedort Ndapeu², Bienvenu Kenmeugne ${ }^{3}$, Tibi Beda ${ }^{4}$ \\ ${ }^{1}$ Department of Physics, Faculty of Sciences, University of Dschang, Dschang, Cameroon \\ ${ }^{2}$ Dschang University/IUT-FV Bandjoun, Dschang, Cameroon \\ ${ }^{3}$ National Advanced School of Engineering (ENSP), Yaounde Department of Industrial and Mechanical Engineering, University of \\ Yaounde I, Yaounde, Cameroon \\ ${ }^{4}$ Department of Physics, Faculty of Sciences, University of Ngaoundere, Ngaoundere, Cameroon \\ Email: nkounhawa@gmail.com
}

How to cite this paper: Nkounhawa, P.K., Ndapeu, D., Kenmeugne, B. and Beda, T. (2020) Analysis of the Behavior of a Square Plate in Free Vibration by FEM in Ansys. World Journal of Mechanics, 10, 11-25. https://doi.org/10.4236/wim.2020.102002

Received: September 28, 2019

Accepted: February 25, 2020

Published: February 28, 2020

Copyright $\odot 2020$ by author(s) and Scientific Research Publishing Inc. This work is licensed under the Creative Commons Attribution International License (CC BY 4.0).

http://creativecommons.org/licenses/by/4.0/

\begin{abstract}
In the realization of mechanical structures, achieving stability and balance is a problem commonly encountered by engineers in the field of civil engineering, mechanics, aeronautics, biomechanics and many others. The study of plate behavior is a very sensitive subject because it is part of the structural elements. The study of the dynamic behavior of free vibration structures is done by modal analysis in order to calculate natural frequencies and modal deformations. In this paper, we present the modal analysis of a thin rectangular plate simply supported. The analytical solution of the differential equation is obtained by applying the method of separating the variables. We are talking about the exact solution of the problem to the limit values. However, numerical methods such as the finite element method allow us to approximate these functions with greater accuracy. It is one of the most powerful computational methods for predicting dynamic response in a complex structure subject to arbitrary boundary conditions. The results obtained by MEF through Ansys 15.0 are then compared with those obtained by the analytical method.
\end{abstract}

\section{Keywords}

Free Vibration, Vibration Modes, Modal Analysis, Natural Frequencies, Modal Deformations, Thin Rectangular Plate, Finite Element Method (FEM)

\section{Introduction}

Structures and buildings are generally subject to increasingly complex excitations. It appears essential to characterize them and then to control their vibratory behavior in order to preserve them against fatigue and rupture [1]. Structural 
failure is generally caused by structural fatigue due to acoustic radiation and amplitude of vibration amplitudes in response to a deterministic or random forced excitation. The increasing use of the plate element in the various industrial sectors, shows the imperative need of the study of their vibrational behavior which thus becomes of great importance in order to help the engineers to design better structures. Vibration analysis is an important current topic, both from an academic and an industrial point of view. The latter affects many areas, such as space technology, naval and civil engineering, automotive, aeronautics, and bridges, buildings, or nuclear engineering. To ensure the desired and desirable mechanical performance in favor of the supporting structure, the determination of the dynamic characteristics of the plates is essential. Rectangular plates are widely used in various engineering disciplines and, from a technical point of view, it becomes necessary to know the natural frequencies of such structures [2].

As the excellent review articles by Leissa [3] [4], Liew, Xiang and Kitipornchai [5] show, vibration analysis is not a recent science. And yet during these decades, it will know a renewed interest for the needs of design and dimensioning of structures responding to the best operating conditions, safety, economy, aesthetics and sound insulation. In addition, it is to optimize, lighten the structures commonly used and subject to significant levels of excitement. For over a hundred years, plaque behavior has been the subject of exhaustive research. It is clear that this research based on the classical Kirchhoff hypothesis neglects the effect of shear deformation and rotational inertia, unlike the Risner-Mindlin plates which take into account these effects.

Born out of the need to solve problems of elasticity and structural analysis involving complex fields of civil engineering and aeronautics, the finite element method was born between the 1960s and the 1970s by the work of the researchers Argyris, Clough, and Zienkiewicz [6] [7]. As a result, several MEFs will be developed for dynamic analysis of structures such as thin and thick plates. Iso parametric elements are the most widely used in plate dynamics [6] [8]. The finite element method is certainly the most favorable because it is one of the most powerful calculation methods for predicting the dynamic response in a complex structure with arbitrary boundary conditions [9].

In this modest work, we are therefore involved in this vast and important field, whose objective is to determine the eigenfrequencies as well as the modes of the vibrations of homogeneous thin isotropic plates in free dynamics by different methods (analytic, finite element method). To treat these vibratory problems, the general idea is to express the deflection of the plate $w$ by a linear combination of the eigen modes. The whole problem then amounts to expressing the $A_{m n}$ coefficients of the proposed form functions. Either we express them experimentally by replacing in the theoretical expression $\mathrm{w}$ by the experimental deflection, or by trying to calculate them theoretically by proposing mathematical models, finally, calculate the matrices of mass and rigidity of the plate and solve the problem with the eigenvalues to obtain the natural frequencies of the plate.

To achieve this, the work done is structured in different sections. Section 2 gives 
a brief description of the kinematics of rectangular plates and governs the equation of motion. In section 3, the study of frequencies and modes of vibration is done by analytical resolution. Section 3 deals with the formulation of dynamic plate behavior by MEF on Ansys. In Section 4, the numerical results are compared to the analytical results for a rectangular plate simply supported on two opposite sides.

\section{Description of the Problem}

We consider a plate having a length $a=1 \mathrm{~m}$, a width $b=1 \mathrm{~m}$ and a thickness $h=$ $0.020 \mathrm{~m}$. The properties of the structure illustrated in Figure 1 are given:

Material: steel;

Poisson coefficient $v$ : 0.3;

Young's modulus $E$ in $a: 2 \times 10^{11}$;

Density $\rho$ in $\mathrm{kg} / \mathrm{m}^{3}: 7850$;

\subsection{Geometry of the Plate}

The plate being simply supported (no displacement and no moment) on two edges, the conditions of fixity make it possible to write:

- No displacement:

$$
\begin{array}{ll}
w(x, y, t)=0 \text { for } x=0, x=a \\
w(x, y, t)=0 \text { for } y=0, y=b
\end{array}
$$

- No moment:

$$
\begin{aligned}
& \frac{\partial^{2} w(x, y, t)}{\partial^{2} x}=0 \text { for } x=0, x=a \\
& \frac{\partial^{2} w(x, y, t)}{\partial^{2} y}=0 \text { for } y=0, y=b
\end{aligned}
$$

\subsection{Fields of Displacement}

In the classical Kirchhoff model, the normal remains straight and is perpendicular to the average surface after deformation. Kirchhoffs field of displacement is then written:

$$
\left\{\begin{array}{l}
u(x, y, t)=u_{0}(x, y)+z \beta_{x}(x, y, t) \\
v(x, y, t)=v_{0}(x, y)+z \beta_{y}(x, y, t) \\
w(x, y, t)=w_{0}(x, y)
\end{array}\right.
$$

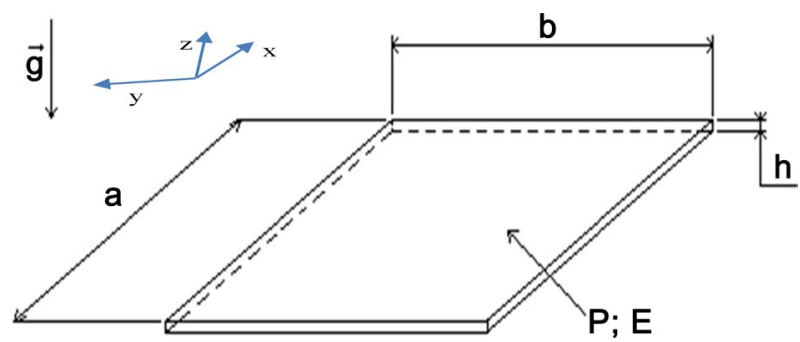

Figure 1. Simply supported rectangular plate and caractéristics. 
$u_{0}$ and $v_{0}$ are displacements of the membrane in $x$ and $y$ direction and $w$ the displacement according to oz or displacement of bending. On the other hand, the rotations are given by:

$$
\begin{gathered}
\beta_{x}=\theta_{y}=-\frac{\partial w}{\partial x} \\
\beta_{y}=-\theta_{x}=-\frac{\partial w}{\partial y} \\
\beta_{z}=0=\frac{\partial w}{\partial z}
\end{gathered}
$$

Which allows to write:

$$
\left\{\begin{array}{l}
u(x, y, t)=u_{0}(x, y)-z \frac{\partial w}{\partial x} \\
v(x, y, t)=v_{0}(x, y)-z \frac{\partial w}{\partial y} \\
w(x, y, t)=w_{0}(x, y)
\end{array}\right.
$$

\subsection{Deformation}

The state of deformation of a plate can be considered as the state of superposition of membrane deformations and flexural deformations. In small deformation, we know that:

$$
\begin{gathered}
\varepsilon_{x x}=-z \frac{\partial^{2} w}{\partial x^{2}}, \quad \varepsilon_{y y}=-z \frac{\partial^{2} w}{\partial y^{2}}, \quad \gamma_{x y}=-z \frac{\partial^{2} w}{\partial x \partial y} \\
\varepsilon_{z z}=\gamma_{x z}=\gamma_{x z}=0
\end{gathered}
$$

What causes these constraints:

$$
\left[\begin{array}{c}
\sigma_{x x} \\
\sigma_{y y} \\
\tau_{x y}
\end{array}\right]=\frac{E}{1-v^{2}}\left[\begin{array}{ccc}
1 & v & 0 \\
v & 1 & 0 \\
0 & 0 & \frac{1-v}{2}
\end{array}\right]\left\{\begin{array}{c}
\varepsilon_{x x} \\
\varepsilon_{y y} \\
\varepsilon_{x y}
\end{array}\right\}
$$

\subsection{Expression of Bending Moments}

The bending moments per unit length in the plate $M x, M y$ and $M x y$ are obtained by integrating the constraints on the thickness of the plate.

$$
M=\int_{-\frac{h}{2}}^{\frac{h}{2}} \sigma z \mathrm{~d} z
$$

\subsection{Definition of Efforts}

Let $N x, N y$ and $N x y$ be these efforts, Hooke allows to write: $N=\sigma S$. Proceeding by integration, we have:

$$
N=\int_{-\frac{h}{2}}^{\frac{h}{2}} \sigma \mathrm{d} z
$$




\subsection{Expression of Deformation and Kinetic Energy}

The deformation energy of the plate is calculated by integration on the volume of the deformation energy density.

$$
u=\int_{v} \sigma \varepsilon \mathrm{d} v=\int_{v} \varepsilon_{i j} c_{i j k l} \varepsilon_{k l} \mathrm{~d} v
$$

The kinetic energy $T$ is calculated by integration of the volume element:

$$
T=\frac{1}{2} \int_{s} m \dot{w}^{2} \mathrm{~d} s
$$

\subsection{Movement Equation}

The vertical equilibrium of the plate element $(\mathrm{d} x, \mathrm{~d} y)$ gives rise to the relation:

$$
\sum \overrightarrow{\text { Fext }}=m \vec{a}
$$

We obtain the differential equation of Lagrange which is a partial derivative equation verified by the vertical displacement:

$$
D\left[\frac{\partial^{4} w}{\partial x^{4}}+\frac{\partial^{4} w}{\partial y^{4}}+2 \frac{\partial^{4} w}{\partial x^{2} \partial y^{2}}\right]-p(x, y)+m \ddot{w}=0
$$

Such vibrations are called free or natural transverse vibrations. As previously stated, the natural vibrations depend only on the properties of the material and the geometry of the plate, and are inherent properties of the elastic plate regardless of any charge. Thus, in the case of free or natural vibration (harmonic movement external load $p(x, y)$ is equal to zero), the only transverse forces acting on the plate are the inertial forces due to mass $\rho$ per unit area, the equation above becomes:

$$
D \nabla^{4} w+\rho h \frac{\partial^{2} w(x, y, t)}{\partial^{2} t}=0
$$

Classical equation of plate theory, which for most technical applications is sufficient for the study of bending problems.

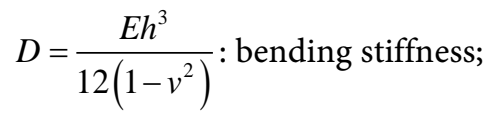

$h$ : thickness of the plate;

$E$ : Young's modulus;

$v$. coefficient of poisson;

$w(x, y, t):$ transverse displacement must satisfy the conditions at the limits of fixity;

\section{Analytical Study of the Rectangular Plate}

The solution to this equation is obtained by looking for the transverse displacement $w(x, y, t)$ such that:

$$
w(x, y, t)=X(x) Y(y) T(t)=w(x, y) \mathrm{e}^{j \omega t}
$$

Which is a solution of the form function. 
$X(x) Y(y)=w(x, y)$ describes the modes of vibration and some harmonic function of a time, $\omega$ is the natural frequency of the vibration of the plate which is related to the frequency and period of the vibration by the relation:

$$
\omega=2 \pi f=\frac{2 \pi}{T}
$$

By introducing these elements, Equation (14) becomes:

$$
\begin{aligned}
& X^{(4)}(x) Y(y) T(t)+2 X^{(2)}(x) Y^{(2)}(y) T(t)+X(x) Y^{(4)}(y) T(t) \\
& =-\frac{\rho h}{D} \cdot X(x) Y(y) T^{(2)}(t)
\end{aligned}
$$

We put: $\frac{D}{\rho h}=\mu^{2}$, and we consider unknown $\beta$

$$
\frac{X^{(4)}(x)}{X(x)}+2 \frac{X^{(2)}(x)}{X(x)} \frac{Y^{(2)}(y)}{Y(y)}+\frac{Y^{(4)}(y)}{Y(y)}=-\frac{1}{\mu^{2}} \frac{T^{(2)}(t)}{T(t)}=\beta^{4}
$$

Which results in:

$$
\left\{\begin{array}{l}
T^{(2)}(t)+\mu^{2} \beta^{4} T(t)=0 \\
\frac{X^{(4)}(x)}{X(x)}+2 \frac{X^{(2)}(x)}{X(x)} \frac{Y^{(2)}(y)}{Y(y)}+\frac{Y^{(4)}(y)}{Y(y)}-\beta^{4}=0
\end{array}\right.
$$

Of these two equations, it follows from the equation of complete displacement of the plate simply supported on two opposite edges:

$$
w(x, y, t)=\sum_{m=1}^{\infty} \sum_{n=1}^{\infty}\left[A_{m n} \cos \omega_{m n} t+B_{m n} \sin \omega_{m n} t\right] \phi_{m n}(x, y)
$$

The coefficients $A_{m n}$ and $B_{m n}$ depending on the load and/or initial conditions. However, the eigen modes are given by:

$$
X_{m}(x) Y_{n}(y)=A_{m n} \cdot \phi_{m n}(x, y)
$$

$w(x, y, t)=0$, represents the modal lines The form function can be taken as:

$$
\begin{aligned}
w(x, y) & =\sum_{m=1}^{\infty} \sum_{n=1}^{\infty} C_{m n} \cdot \phi_{m n}(x, y) \\
& =\sum_{m=1}^{\infty} \sum_{n=1}^{\infty} C_{m n} \cdot \sin \frac{m \pi}{l_{x}} x \cdot \sin \frac{n \pi}{l_{y}} y
\end{aligned}
$$

$\phi_{m n}(x, y)=\sin \frac{m \pi}{l_{x}} x \cdot \sin \frac{n \pi}{l_{y}} y$ represents the modal deformed, the own deformed, of the $\mathrm{nm}$ mode satisfying the supported boundary conditions, $\mathrm{a}$ and $\mathrm{b}$ are respectively the length and the width of the plate, $C_{m n}$ are the modal coefficients, corresponding to the projection of the motion in the modal base. This is the vibration amplitude for each value of $m$ and $n$.

By replacing the expression

$$
w(x, y)=\sum_{m=1}^{\infty} \sum_{n=1}^{\infty} A_{m n} \sin \frac{m \pi}{l_{x}} x \cdot \sin \frac{n \pi}{l_{y}} y
$$

In the main Equation (14), we obtain: 


$$
\frac{m^{4} \pi^{4}}{a^{4}}+2 \frac{m^{2} \pi^{2}}{a^{2}} \frac{n^{2} \pi^{2}}{b^{2}}+\frac{n^{4} \pi^{4}}{b^{4}}-\omega^{2} \frac{\rho h}{D}=0
$$

The resolution of this equation leads to a natural pulse $\omega_{m n}$ of the mode $\mathrm{m}$ and $\mathrm{n}$ such that:

$$
\omega_{m n}=\pi \sqrt{\frac{D}{\rho h}} \cdot\left[\left(\frac{n}{l_{y}}\right)^{2}+\left(\frac{m}{l_{x}}\right)^{2}\right]
$$

Eigen frequency: $f_{m n}=\frac{\omega_{m n}}{2 \pi}$

In the case of the plate under study, we have:

$$
D=\frac{E h^{3}}{12\left(1-v^{2}\right)}=146520.15 \mathrm{~N} \cdot \mathrm{m}^{2},
$$

The analysis of the first two modes gives us:

- if $m=1, n=1, \omega_{11}=\frac{\pi^{2}}{L^{2}}\left[1^{2}+1^{2}\right] \sqrt{\frac{D}{\rho h}}=\frac{\pi^{2}}{L^{2}}[2] \sqrt{\frac{D}{\rho h}}, \omega_{11}=603.02 \mathrm{rad} / \mathrm{s}$

and $f_{11}=\frac{\omega_{11}}{2 \pi}=95.97 \mathrm{~Hz}$

- if $m=1, n=2, \omega_{21}=\omega_{12}=\frac{\pi^{2}}{L^{2}}[5] \sqrt{\frac{D}{\rho h}}=1507.53 \mathrm{rad} / \mathrm{s}$ and

$$
f_{21}=f_{12}=\frac{\omega_{21}}{2 \pi}=239.93 \mathrm{~Hz}
$$

The analysis of natural pulsations by the Rayleigh technique confirms the veracity of these results especially for the first vibratory mode.

\section{Modeling of the Dynamic Behavior of Plates by FEM in Ansys}

The finite element method is a widely used and powerful tool for the analysis of complex structures. It consists of a discretization of the element into a finite number of generally triangular or rectangular elements [10]. This resolution technique involves the calculation of the matrix of mass and rigidity of the whole structure:

$$
[K]-\omega^{2}[M]=0
$$

Equation (24) will be solved to give the eigenfrequencies and the eigen mode or modal deformations of the structure using the ANSYS software for a simply supported plate.

Let us always consider our rectangular plate whose characteristics are defined in section 1 and represented under Ansys in Figure 2. The plate is modeled on a volume of 2, e $+007 \mathrm{~mm}^{3}$ and has a mass of $157 \mathrm{~kg}$.

The dimensions of the plate illustrated in Figure 2 and Figure 3 are given in section 2. The simulations are made on the plate having a referential temperature of $22^{\circ} \mathrm{C}$ and discretized in 2401 elements for 17,300 nodes as seen in Figure 3. The bar graph indicates the specific frequency of each calculated mode and 
whose values are given in Table 1 after modal analysis of the structure.

It is noted that the frequencies grow with vibratory modes as illustrated in Figure 4 and Figure 5. On the other hand the displacements of the plate as shown in Figure 6 do not follow this law, they oscillate around $6 \mathrm{~mm}$.

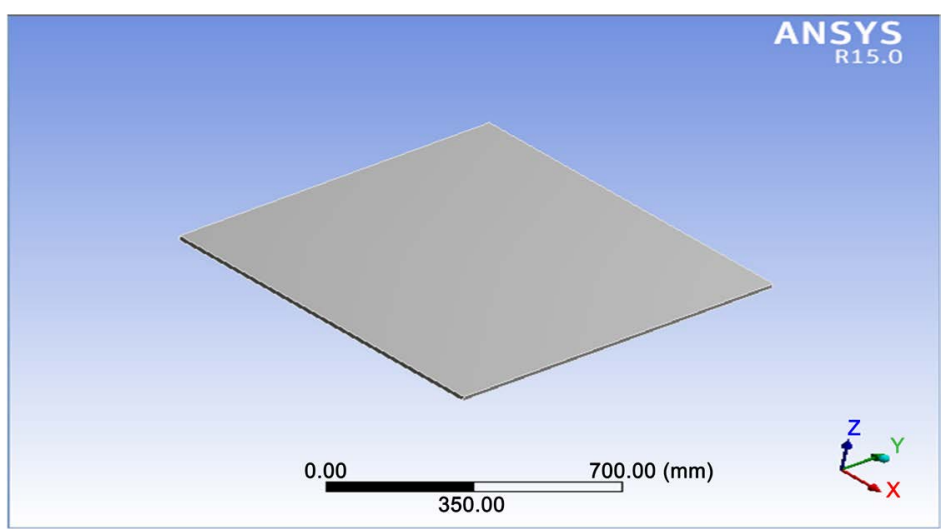

Figure 2. Plate geometry under Ansys.

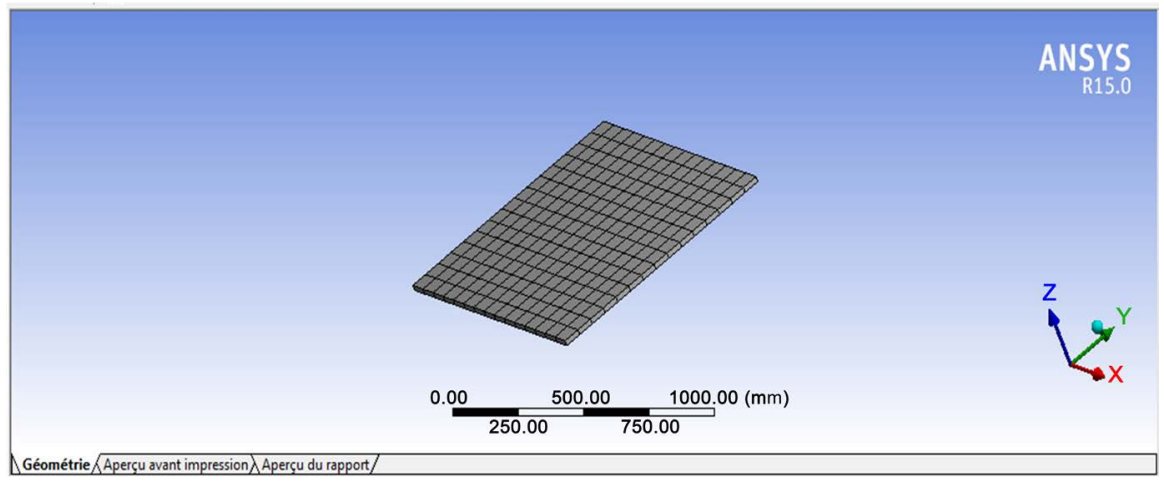

Figure 3. Mesh of the plate.

Table 1. Frequency values for different plate modes.

\begin{tabular}{lccc}
\hline & Mode & Frequency $[\mathrm{Hz}]$ & Max displacement $[\mathrm{mm}]$ \\
\hline $\mathbf{1}$ & $m=1, n=1$ & 107.9 & 4.5143 \\
$\mathbf{2}$ & $m=2, n=1$ & 128.1 & 6.8385 \\
$\mathbf{3}$ & $\mathrm{m}=2, n=1$ & 210.6 & 7.4719 \\
$\mathbf{4}$ & $m=1, n=2$ & 296.74 & 4.556 \\
$\mathbf{5}$ & $m=2, n=2$ & 325.11 & 6.5421 \\
$\mathbf{6}$ & $m=3, n=1$ & 384.8 & 7.548 \\
7 & $m=3, n=2$ & 422.25 & 6.8763 \\
$\mathbf{8}$ & $m=1, n=3$ & 579.94 & 4.7202 \\
9 & $m=3, n=2$ & 597.47 & 7.2271 \\
10 & $m=2, n=3$ & 610.77 & 6.5952 \\
11 & $m=3, n=1$ & 658.27 & 7.3154 \\
12 & $m=3, n=3$ & 711.4 & 6.6124 \\
\hline
\end{tabular}




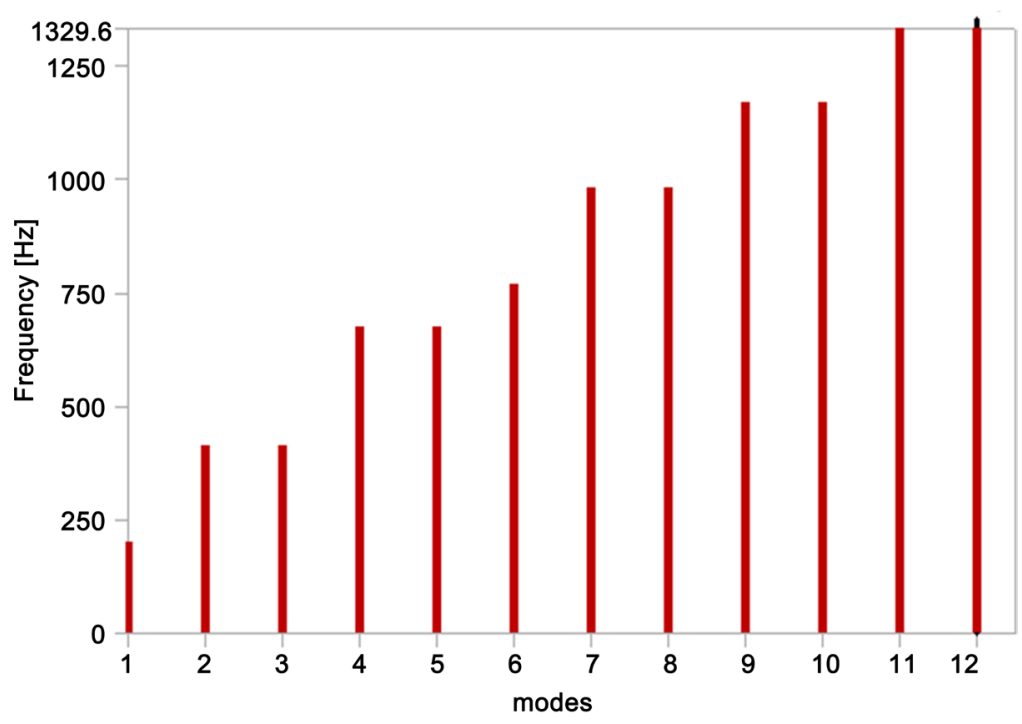

Figure 4. Frequency evolution.

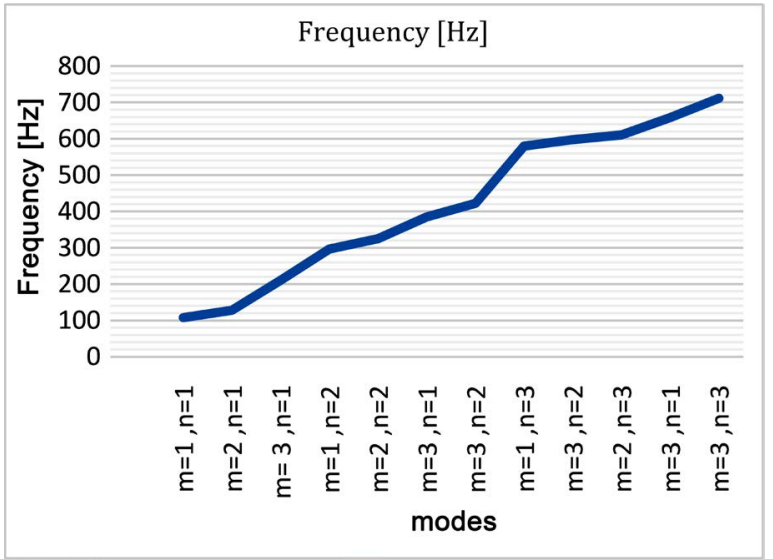

Figure 5. Specific frequency of each mode.

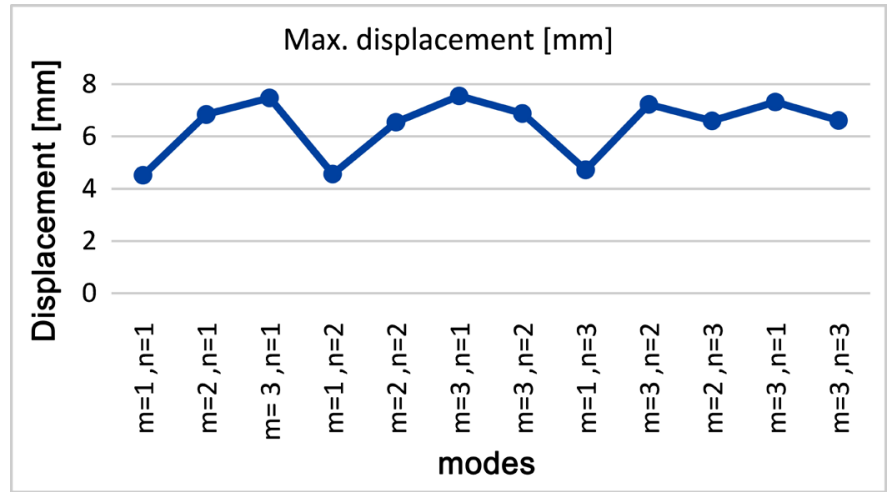

Figure 6. Modal displacement of the plate.

As shown in Figure 6, the maximum displacement is observed when $m=3, n$ $=1$ and is equal to $7.548 \mathrm{~mm}$.

These frequencies are geometrically translated by the following modes of Figure 7 (from (a) to (l)): 

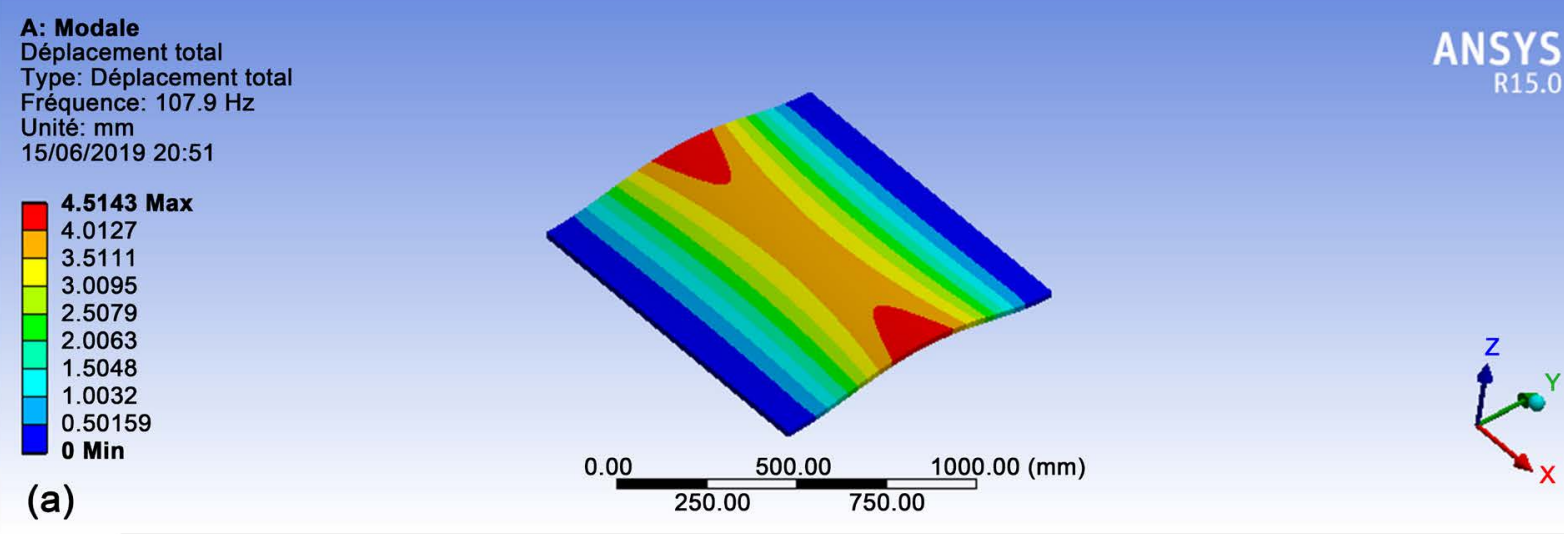

\section{A: Modale}

Déplacement total 2

Type: Déplacement total

Fréquence: $128.1 \mathrm{~Hz}$

Unité: $\mathrm{mm}$

15/06/2019 20:51

\begin{tabular}{|l|l|}
6.8385 & Max \\
6.0787 \\
5.3188 \\
4.559 \\
3.7992 \\
3.0393 \\
2.2795 \\
1.5197 \\
0.75983 \\
O Min
\end{tabular}

(b)

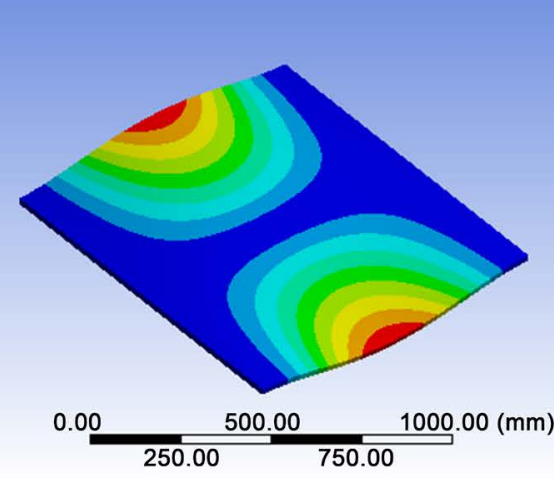

\section{ANSYS}

R15.0

\section{A: Modale \\ Déplacement total 3 \\ Unité: $\mathrm{mm}$ \\ 15/06/2019 20:50

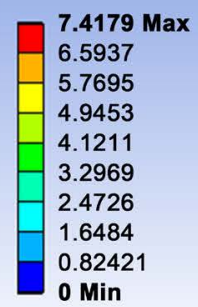

Type: Déplacement tota

Fréquence: $210.6 \mathrm{~Hz}$

(c)

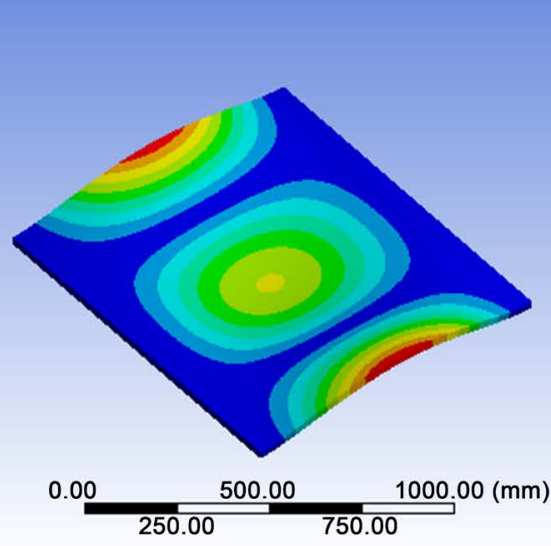

\section{ANSYS}

R15.0

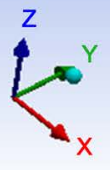

\section{A: Modale}

Déplacement total 4

Type: Déplacement tota

Fréquence: $296.74 \mathrm{~Hz}$

Unité: $\mathrm{mm}$

15/06/2019 20:49
$4.556 \mathrm{Max}$
4.0498
3.5436
3.0374
2.5311
2.0249
1.5187
1.0125
0.50623

(d)

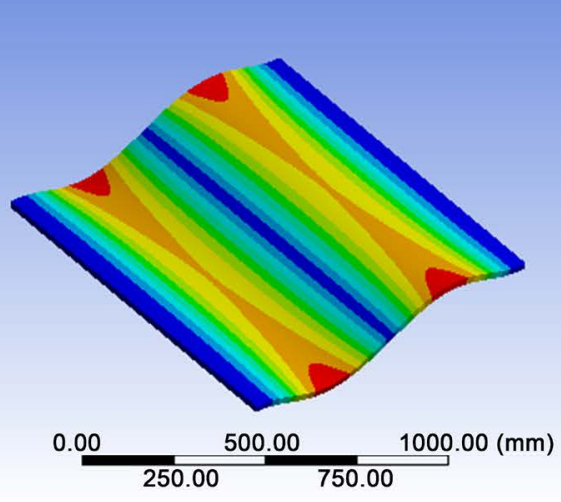

ANSYS

R15.0

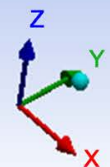




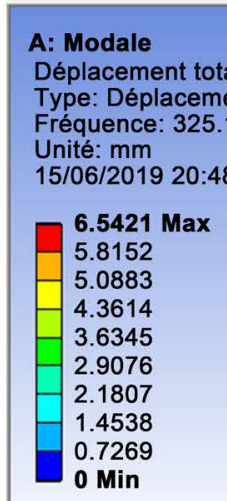

(e)

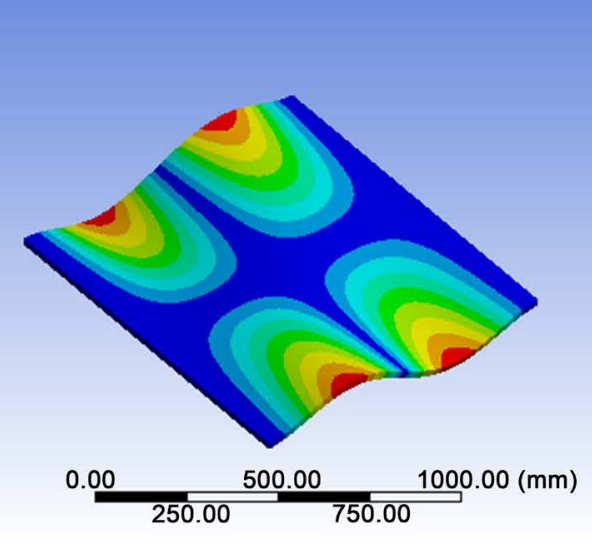

ANSYS

R15.0

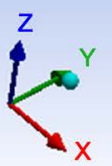

ANSYS

R15.0

Déplacement total 6

Type: Déplacement total

Fréquence: $384.8 \mathrm{~Hz}$

Unité: $\mathrm{mm}$

15/06/2019 20:47
7.548 Max
6.7093
5.8707
5.032
4.1933
3.3547
2.516
1.6773
0.83867

(f)
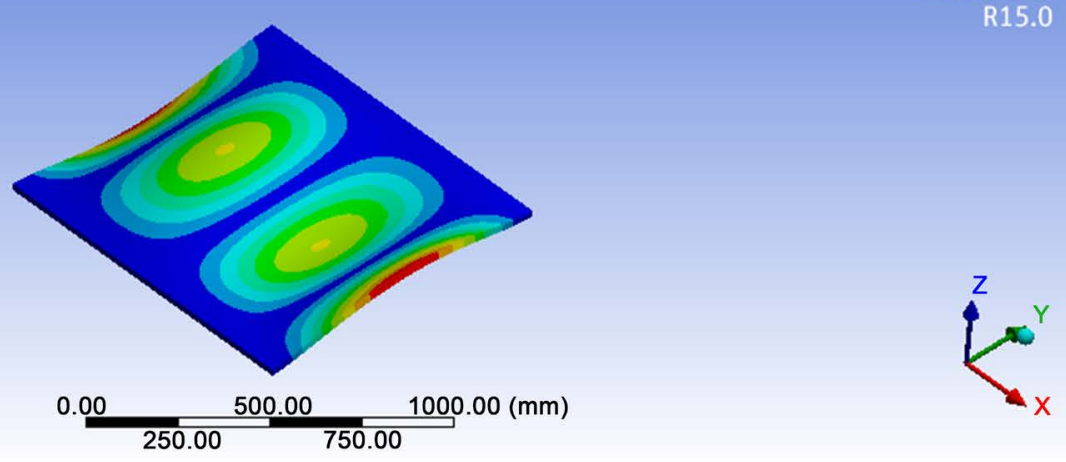

A: Modale

Déplacement total 7

Type: Déplacement tota

Fréquence: $422.25 \mathrm{~Hz}$

Unité: $\mathrm{mm}$

15/06/2019 20:47

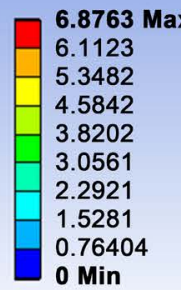

(g)

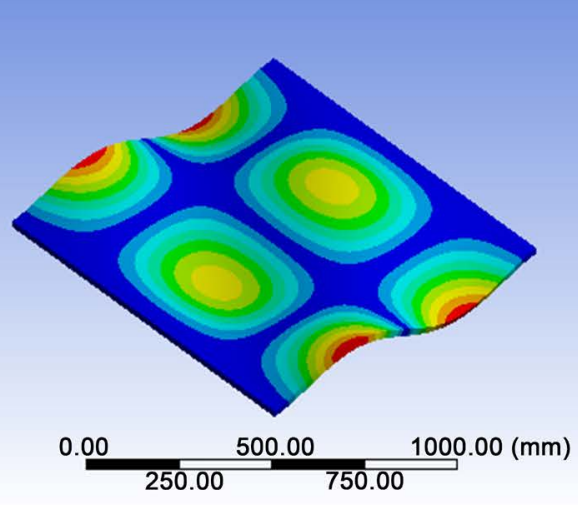

ANSYS

R15.0

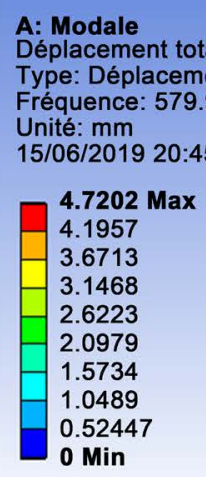

(h)

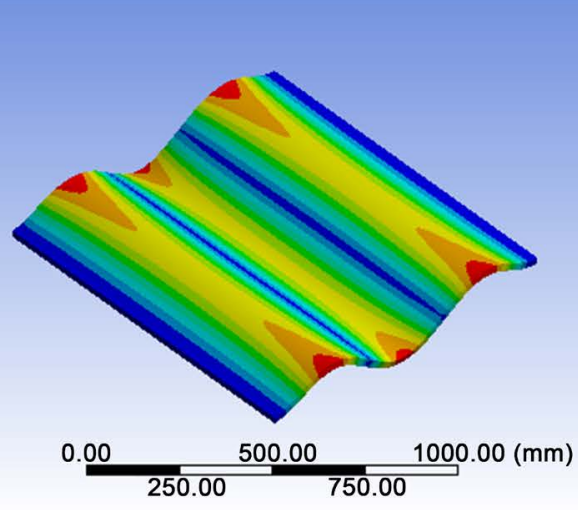

ANSYS 

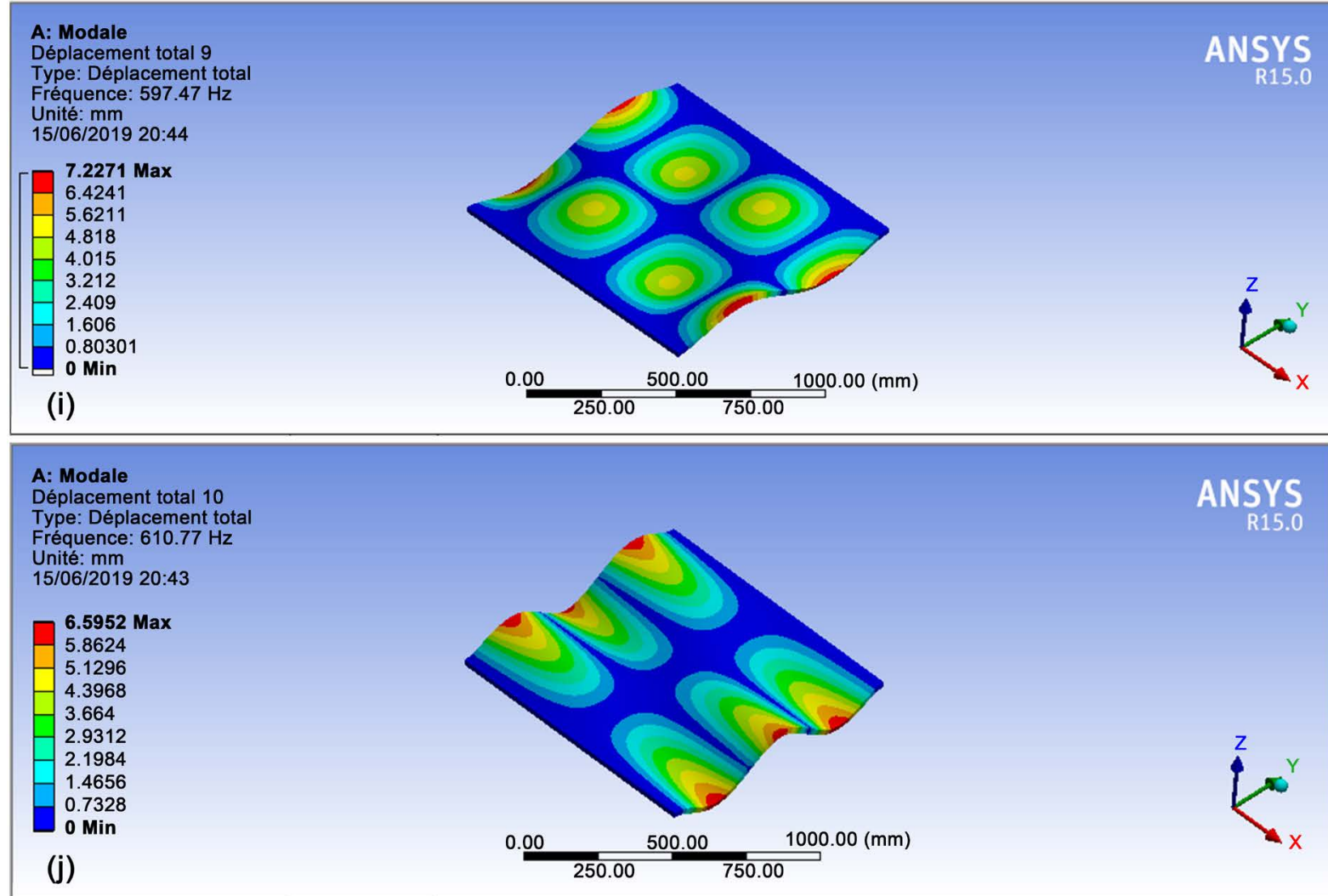

ANSYS

Type: Déplacement total

réquence: $610.77 \mathrm{~Hz}$

5/06/2019 20:43

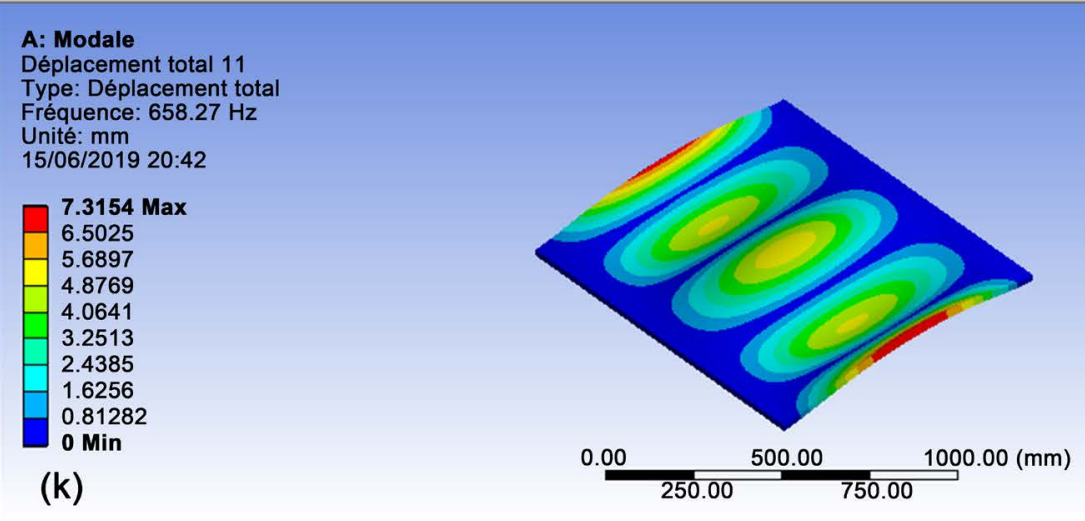

ANSYS

R15.0

A: Modale

Déplacement total 12

Type: Déplacement total

Fréquence: $717.4 \mathrm{~Hz}$

Unité: $\mathrm{mm}$

15/06/2019 20:41

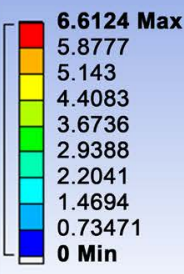

(I)
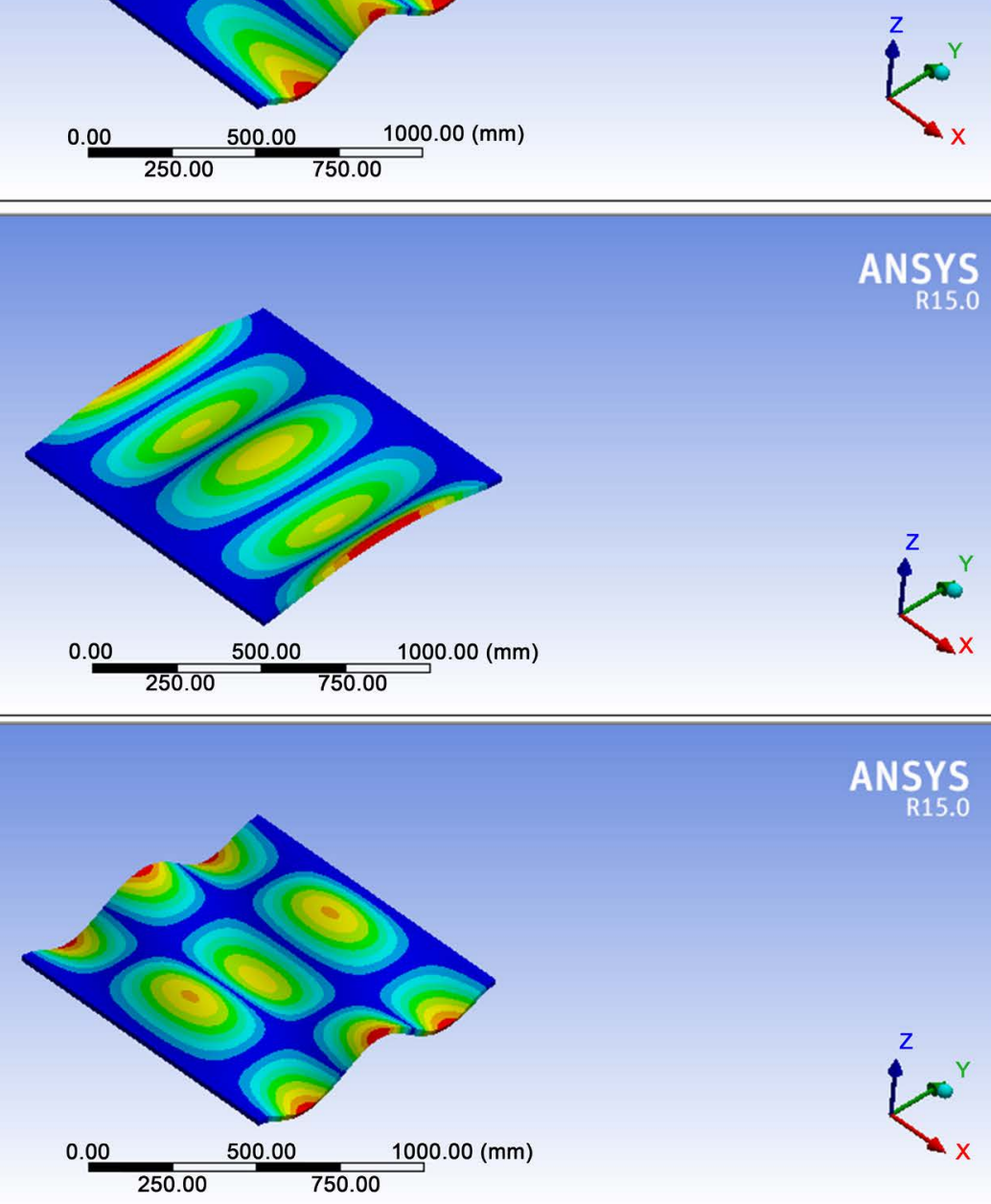

Figure 7. Vibratory modes of a plate simply supported on two opposite edges (from (a) to (l)). (a) mode $1: m=1, n=1 ;$ (b) mode $2: m$ $=2, n=1$; (c) mode 3: $m=2, n=1$; (d) mode 4: $m=1, n=2$; (e) mode 5: $m=2, n=2$; (f) mode 6: $m=3, n=1 ;$ (g) mode 7: $m=3, n=$ 2; (h) mode 8: $m=1, n=3$; (i) mode 9: $m=3, n=2$; (j) mode 10: $m=2, n=3$; (k) mode 11: $m=3$; $n=1$; (l) mode $12: m=3, n=3$. 


\section{Comparison of the Eigenfrequencies Analytic and Those Obtained by Finite Elements}

Table 1 and Table 2 show the values of the eigenfrequencies obtained by two methods considered, analytical and finite element structure calculation, corresponding to the rectangular configuration. From these two tables and in accordance with Figure 8 and Figure 9, we use Equation (25) to calculate the error of the modes $m=1, n=1 ; m=1, n=2 ; m=2, n=2 ; m=3^{\prime}, n=1$ and $m=3, n=3$ :

$$
\varepsilon(\%)=\frac{f S-f C}{f C}
$$

Table 2. Comparison of the eigenfrequencies analytic and those obtained by finite elements.

\begin{tabular}{cccccc}
\hline Mode & $\begin{array}{c}\text { modal } \\
\text { deformation }\end{array}$ & $\begin{array}{c}\text { Frequency } \\
\text { MEF [Hz] }\end{array}$ & $\begin{array}{c}\text { Calculated Frequency } \\
{[\mathrm{Hz}]}\end{array}$ & Gap (Hz) & $\boldsymbol{\varepsilon}(\%)$ \\
\hline 1 & $m=1, n=1$ & 107.9 & 95.97 & 11.93 & 0.1243 \\
4 & $m=1, n=2$ & 296.74 & 239.93 & 56.81 & 0.2367 \\
5 & $m=2, n=2$ & 325.11 & 383.89 & -58.78 & 0.1531 \\
11 & $m=3, n=1$ & 658.27 & 623.82 & 34.45 & 0.0552 \\
12 & $m=3, n=3$ & 711.4 & 863.75 & -152.35 & 0.1763 \\
\hline
\end{tabular}

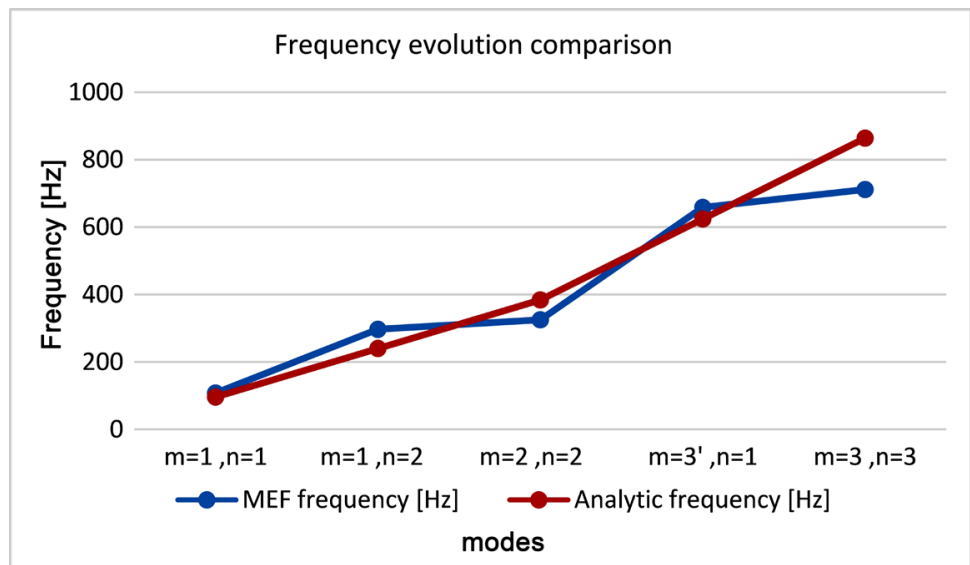

Figure 8. Comparison between MEF and calculated frequency.

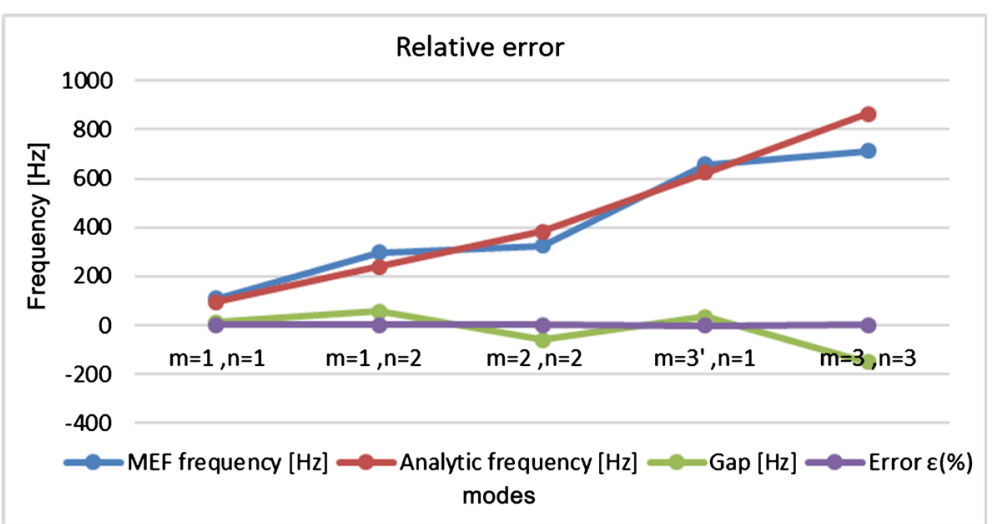

Figure 9. Error graph. 
The error between the two methods use in this case study is minimized and converges to zero according to the error graph illustrated in Figure 9.

\section{Conclusion}

In this paper, we highlight the dynamic analysis of a plate simply supported on two opposite edges in free vibration. Two techniques have been deployed to approach the fundamental eigenfrequencies of the plate to be studied. It is the method of separable variables based on the modeling of the transversal displacement from the characteristic functions of vibrations of the thin plates and the analysis by the method of the finite elements. The responses obtained analytically and numerically from the nonlinear equations developed for the calculation of the eigenvalues of the plate show us that the frequencies increase with the modal deformations. The conditions of fixity and their location impose a great influence on the behavior of the plate structure in vibration. A comparison between the frequencies calculated and those obtained by Ansys for the rectangular plates reveals a convergence of the two calculation techniques.

\section{Conflicts of Interest}

The authors declare no conflicts of interest regarding the publication of this paper.

\section{References}

[1] Nowacki, W. (1963) Dynamics of Elastic Systems. John Wiley and Sons, New York.

[2] Kalita, K. and Haldar, S. (2016) Free Vibration Analysis of Rectangular Plates with Central Cutout. Cogent Engineering, 3, 1163781. https://doi.org/10.1080/23311916.2016.1163781

[3] Leissa, A.W. (1978) Recent Research in Plate Vibrations, 1973-1976: Classical Theory. Shock and Vibration Inform. Center the Shock and Vibration Digest, 9, 13-24. https://doi.org/10.1177/058310247700901005

[4] Leissa, A.W. (1987) Literature Review: Survey and Analysis of the Shock and Vibration Literature: Recent Studies in Plate Vibrations: 1981-85 Part I. Classical Theory. The Shock and Vibration Digest, 19, 11-18. https://doi.org/10.1177/058310248701900204

[5] Liew, K.M., Xiang, Y. and Kitipornchai, S. (1993) Transverse Vibration of Thick Rectangular Plates-I. Comprehensive Sets of Boundary Conditions. Computers \& Structures, 49, 1-29. https://doi.org/10.1016/0045-7949(93)90122-T

[6] Clough, R.W. and Tocher, J.L. (1965) Finite Element Stiffness Matrices for Analysis of Plates in Bending. Proceedings of Conference on Matrix Methods in Structural Analysis, 1, 515-545.

[7] Zienkiewicz, O.C. and Taylor, R.L. (2005) The Finite Element Method for Solid and Structural Mechanics. Butterworth-Heinemann, Oxford.

[8] Batoz, J.L., Bathe, K.J. and Ho, L.W. (1980) A Study of Three-Node Triangular Plate Bending Elements. International Journal for Numerical Methods in Engineering, 15, 1771-1812. https://doi.org/10.1002/nme.1620151205

[9] Park, I., Lee, U. and Park, D. (2015) Transverse Vibration of the Thin Plates: Fre- 
quency-Domain Spectral Element Modeling and Analysis. Mathematical Problems in Engineering, 2015, Article ID 541276. https://doi.org/10.1155/2015/541276

[10] Pouladkhan, A.R., Emadi, J., Safamehr, M. and Habibolahiyan, H. (2011) The Vibration of Thin Plates by Using Modal Analysis. World Academy of Science, Engineering and Technology, 59, 2880-2885. 